\title{
Altered Cell Adhesion and Glycosylation Promote Cancer Immune Suppression and Metastasis
}

\author{
Heinz Läubli ${ }^{1 \dagger}$ and Lubor Borsig ${ }^{2,3 * \dagger}$ \\ ${ }^{1}$ Laboratory for Cancer Immunotherapy, Department of Biomedicine and Medical Oncology, Department of Internal \\ Medicine, University Hospital, Basel, Switzerland, ${ }^{2}$ Department of Physiology, University of Zurich, Zurich, Switzerland, \\ ${ }^{3}$ Comprehensive Cancer Center, Zurich, Switzerland
}

OPEN ACCESS

Edited by:

Toshiyuki Murai,

Osaka University, Japan

Reviewed by:

Janko Kos,

University of Ljubljana, Slovenia

Fabrice Cognasse,

Groupe Sur L'immunité Des Muqueuses Et Agents Pathogènes

(GIMAP), France

*Correspondence:

Lubor Borsig

Iborsig@access.uzh.ch

tThese authors have contributed equally to this work

Specialty section:

This article was submitted to Cancer Immunity and Immunotherapy,

a section of the journal

Frontiers in Immunology

Received: 14 July 2019

Accepted: 23 August 2019

Published: 06 September 2019

Citation:

Läubli H and Borsig L (2019) Altered

Cell Adhesion and Glycosylation

Promote Cancer Immune Suppression

and Metastasis.

Front. Immunol. 10:2120.

doi: 10.3389/fimmu.2019.02120
Cell-cell interactions and cell adhesion are key mediators of cancer progression and facilitate hallmarks of cancer including immune evasion and metastatic dissemination. Many cell adhesion molecules within the tumor microenvironment are changed and significant alterations of glycosylation are observed. These changes in cell adhesion molecules alter the ability of tumor cells to interact with other cells and extracellular matrix proteins. Three families of cell-cell interaction molecules selectins, Siglecs, and integrins have been associated with cancer progression in many pre-clinical studies, yet inhibition of cell adhesion as a therapeutic target is just beginning to be explored. We review how cell-cell interactions mediated by integrins and the glycan-binding receptors selectins and Siglec receptors support cancer progression. The discussion focuses on mechanisms during immune evasion and metastasis that can be therapeutically targeted by blocking these cell-cell interactions.

Keywords: selectin, Siglec, integrin, immunity, sialic acid, tumor microenvironment

\section{INTRODUCTION}

Cancer progression induces immune evasion and eventually metastasis, a process consisting of several steps enabling tumor cells to leave the primary tumor, to intravasate and survive in the circulation, to extravasate and seed in distant organs and to initiate growth of metastatic lesions. Tumor cell interactions with other cells in the environment contribute to immune evasion and metastasis at every step of this process. Adhesion molecules, on any cell, mediate interactions with other cells and the extracellular matrix in the microenvironment $(1,2)$. Since cell adhesion receptors are connected to signal-transduction pathways, these cell-cell and cell-matrix interactions modulate cell phenotype, proliferation, differentiation, survival, and migration. Consequently, changes in expression of cell adhesion molecules and their ligands directly affect immune evasion and metastasis.

Malignant transformation changes not only the expression of cell adhesion molecules but also causes profound changes in cell surface glycosylation $(3,4)$. Cancer-associated glycosylation promotes the interaction of tumor cells within a microenvironment through glycan-binding receptors-lectins (5). Glycans are oligosaccharide structures presented on protein and lipids. Endogenous lectins expressed on immune cells and other cells in the stroma, facilitate cell-interactions, -adhesions, thereby contributing to homeostasis. During malignancy, glycans on tumor cells are involved in invasiveness, metastasis, and immune suppression (6-8).

Several families of cell adhesion molecules including cadherins, integrins, junctional-adhesion molecules, and selectins are altered during tumorigenesis. This mini review addresses the role of 
cell adhesion and glycan-mediated interactions during metastasis and tumor-induced immune suppression in the context of altered glycosylation as a ubiquitous characteristic of cancer progression with a focus on integrins, selectins, and siglecs.

\section{SELECTINS CONTRIBUTE TO CANCER PROGRESSION}

Selectins are vascular cell adhesion receptors present on leukocytes, endothelial cells, and platelets that bind to glycans. The physiological function of selectins is to facilitate the initial tethering of leukocytes at inflammatory sites or secondary lymphoid organs or hemostasis $(9,10)$. There is accumulating evidence for the involvement of selectins in pathophysiological processes, including cancer metastasis $(11,12)$. There are three members of the selectin family: P-selectin expressed on activated platelets and endothelial cells, L-selectin present on leukocytes, and E-selectin expressed on activated endothelial cells (10). Upon activation, P-selectin is rapidly presented on the surface of activated endothelial cells or platelets through exocytosis of storage granules. E-selectin is present only on activated endothelial cells and its expression is regulated on a transcription level. L-selectin is constitutively expressed on most subsets of leukocytes.
Selectins are C-type lectins that bind to properly modified glycan ligands, carrying terminal sLe $e^{\mathrm{x}}$ or sLe $\mathrm{a}^{\mathrm{a}}$ structures. Selectin binding to glycans usually requires a protein scaffold that presents selectin ligands in clusters (13). The best characterized selectin ligand is P-selectin glycoprotein ligand 1 (PSGL-1) (9, 10). All three selectins bind to PSGL-1 that is mostly expressed in leukocytes. In addition, selectins binds to these glycan moieties carried on several cell surface proteins, such as CD44, Eselectin ligand-1, CD43, CD34, or addressins with a variable specificity (14).

There is compelling experimental and clinical evidence for the enhanced expression of $\mathrm{sLe}^{\mathrm{x}}$ and $\mathrm{sLe}^{\mathrm{a}}$ to correlate with poor prognosis due to enhanced metastasis in tumors of gastric, pancreatic, colon, prostate, renal, lung, and melanoma cancers $(4,15)$. Enhanced expression of selectin ligands is linked to increased activities of glycosyltransferases, responsible for the terminal synthesis, sialyltransferases, and fucosyltransferases. Major carriers of selectin ligands are mucins that are heavily O-glycosylated (16). MUC1, MUC2, MUC4, and MUC16 are mucins associated with cancer progression, whereas MUC16 is also used for cancer diagnostics. However, the spectrum of selectin ligands on tumor cells is rather broad, encompassing glycolipids, proteins, and glycosaminoglycans (4).

During the hematogenous phase of metastasis, tumor cells carrying selectin ligands (Figure 1) enter the blood circulation

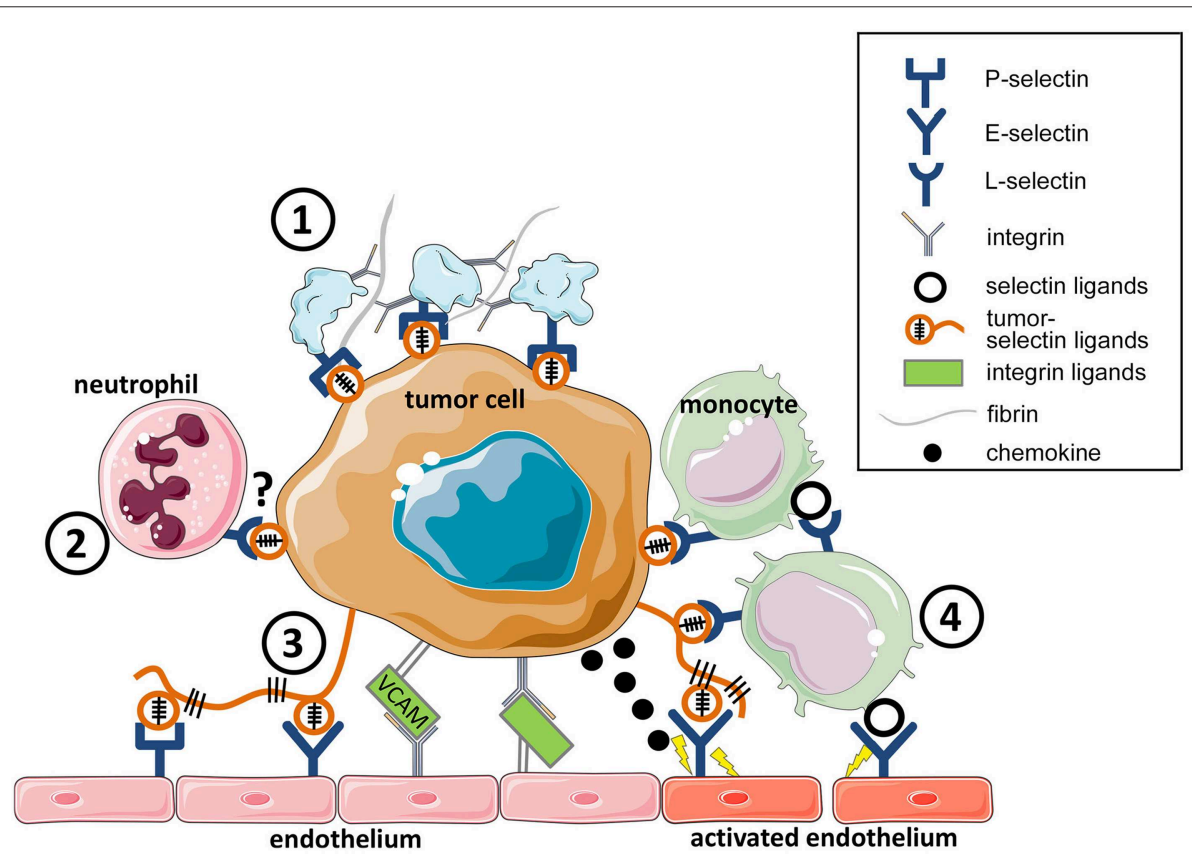

FIGURE 1 | Cell adhesion facilitates tumor cell survival in the circulation and tumor cell extravasation. Tumor cells in the circulation interact through selectins and integrins with blood constituents (platelets, leukocytes, and endothelial cells). (1) platelet-tumor cell aggregate formation is mediated by both P-selectin and integrins through fibrin and fibrinogen. (2) The survival of circulating tumor cells is further enhanced by aggregation with neutrophils that promote tumor cell proliferation (17). Whether L-selectin or integrins mediate these interactions remains to be determined. (3) Tumor cell interaction with the endothelium, leading to adherence, is mediated by P-and E-selectins, and tumor cell firm adhesion is facilitated by integrins, and their interaction, for example with VCAM-1 on tumor cells. (4) Tumor cell-endothelial interaction directly or facilitated by monocytes, contribute to the initiation of tumor cell extravasation. This process is dependent both on E-selectin and integrin engagement. 
and encounter selectins on platelets, leukocytes, and on the endothelium (14). Tumor cells in the circulation are often associated with platelets that protects them from the immune system (18), and enables tumor cell seeding in distant organs (19). The absence of P-selectin abrogates platelet-tumor cell aggregation and consequently attenuates metastasis (20). In addition to P-selectin, platelets also express CD40 on their surfaces, which upon binding to the CD40 ligand accelerate endothelial inflammation and atherosclerosis (21). Plateletleukocyte aggregate formation resulted in a release of IL- $1 \beta$ by leukocytes. CD40 deficiency in the blood compartment attenuated experimental lung metastasis (22), indicating a potential involvement of platelet CD40 in cancer progression. Tumor cell arrest in the vasculature induces local activation of the endothelium and results in expression of E-selectin and chemokines $(12,23)$. Chemokine-driven recruitment of inflammatory monocytes to metastasizing cells was shown to promote metastasis in different cancers [reviewed in (24)]. E-selectin has been shown to promote tumor cell adhesion and thereby metastatic dissemination $(14,25,26)$. Enhanced expression of E-selectin ligands on human breast cancer cells, such as CD44, promotes homing to the microvascular endothelium and metastasis (27).

Selectins not only mediate tumor cell adhesion but actively contribute to the formation of a metastasis niche (28, 29). Effective tumor cell extravasation in the lungs requires engagement of E-selectin on activated endothelial cells, which is essential for the loosening of endothelial VE-cadherin junctions (28). E-selectin promotes the recruitment of inflammatory monocytes, Ly6 $\mathrm{C}^{\text {hi }}$ cells that facilitate transendothelial migration of tumor cells (Figure 1). The opening of endothelial junctions was shown to be dependent on the Src kinase pathway that induces E-selectin expression (30). Recently, E-selectin in the bone marrow vascular niche was shown to promote metastasis by inducing mesenchymal-epithelial transition of breast cancer cells through the activation of Wnt signaling (29). Tumor cell expression of fucosyltransferase-7, required for E-selectin ligand formation, is essential for the formation of bone metastasis.

L-selectin-mediated recruitment of leukocytes promotes both tumor cell extravasation and the formation of a metastatic niche $(28,31,32)$. Tumor-induced endothelial activation is associated with selectin ligand accumulation required for the L-selectin dependent recruitment of myeloid cells (31), which were later identified to be inflammatory monocyte Ly $6 \mathrm{C}^{\text {hi }}$ cells $(33,34)$. Interestingly, the presence of selectin ligands on leukocytes is also required for their effective recruitment to the metastatic sites (34). L-selectin facilitates the recruitment of T-cells to the lymph nodes. An engagement of a $\mathrm{T}$ cell receptor leads to the shedding of L-selectin from the cell surface of $\mathrm{T}$ cells $(35,36)$. Notably, cytotoxic/memory T cells, with L-selectin expression, better controlled tumor growth (37, 38). Sustained L-selectin expression on NK cells was shown to control tumor progression (39). Recently, the role of Lselectin on $\mathrm{T}$ cells was investigated in a transgenic mouse model that expressed non-cleavable L-selectin (40). T cells with constitutive expression of L-selectin suppressed lung metastasis. However, how L-selectin on $\mathrm{T}$ and $\mathrm{NK}$ cells contributes to the immune suppressive activity during metastasis remains to be defined.

\section{SIGLEC-MEDIATED IMMUNE SUPPRESSION IN CANCER}

The cell surface glycans of mammalian cells commonly terminate with sialic acid (41). These sialylated structures, also called sialoglycan can engage various endogenous receptors including sialic acid-binding immunoglobulin-like lectins (Siglecs) (7, 4244). Siglecs are mostly inhibitory receptors with an extracellular part that contains an $\mathrm{N}$-terminal carbohydrate recognition domain (CRD) and a variable number of C2 domains (44). The intracellular part of inhibitory Siglecs contains ITIM or ITIM-like structures mediating immune inhibition (7, 42-44). Activating Siglecs have a positively charged amino acid in the transmembrane domain that mediates interaction with DAP12 upon Siglec engagement $(7,42-44)$. In humans, 14 different, functionally active Siglecs were identified. The conserved Siglecs Siglec-1 (sialoadhesin), CD22 (Siglec-2), Siglec-4 (MAG), and Siglec-15 have orthologs across different mammalian species $(45,46)$. CD33-related Siglecs, however, have undergone rapid evolutionary adaptation $(45,46)$. This subfamily includes CD33 (Siglec-3), Siglec-5, Siglec-6, Siglec-7, Siglec-8, Siglec-9, Siglec-10, and Siglec-11 $(45,46)$. In mice, no direct orthologs of human CD33-related Siglecs can be found, but functional paralogues with similar expression patterns can be defined (47).

Siglec receptors are predominantly expressed on immune cells (7, 42-44). Inhibitory Siglec receptors can modulate immune cell activation by recruitment of SHP1 and SHP2 phosphatase upon binding to sialoglycans (7, 42-44). Binding-specificity varies between different Siglecs. While Siglec-9 has quite a broad binding spectrum $(47,48)$, Siglec- 8 binds a rather restricted set of sialoglycans which also contain sulfate groups $(49,50)$. The binding spectrum and the expression patterns determine the function of Siglecs.

Recent evidence has shown that tumor cells can also engage the sialoglycan-Siglec axis to evade immune control $(7,47$, 51-56). In many cancer types, the glycocalyx and also the tumor microenvironment are characterized by an enhanced presence of sialoglycans due to changes in sialic acid-modifying enzymes including sialic acid synthesis genes, transporters, sialyltransferases, and sialidases (57-59). Moreover, enzymes such as $\mathrm{O}$-acetylases can directly modify the sialic acid residues (57-59). This upregulation of sialoglycans in some cancers is termed hypersialylation, which is quite heterogenous between different cancer types but also within a specific cancer type. In lung cancer, we have observed considerable heterogeneity of sialoglycan ligands for Siglec-7 and Siglec-9 (51). Similar observations were made in melanoma samples (52). How sialylation differs within a single cancer patient and how hypersialylation evolves during different treatments and during cancer progression over time remains to be determined.

The increased density of sialoglycans can lead to engagement of inhibitory Siglec receptors on immune cells and modulate the immune response to cancer (Figure 2). Both innate and 
adaptive immune cells can be regulated by the sialoglycanSiglec checkpoint. Human NK cells express inhibitory Siglec7 and some subpopulations of NK cells also express Siglec-9 $(54,55)$. Engagement of Siglec-7 and/or Siglec-9 can inhibit NK cell-mediated tumor cell killing in vitro $(54,55)$. The introduction of a synthetic sialoglycan polymer into the glycocalyx of target cells led to a significant decrease in the NK cell-mediated killing of cells lacking MHC I expression and a reduced antibody-dependent cellular cytotoxicity (54). Antibodies blocking Siglec-7 or Siglec-9 resulted in increased tumor cell killing (55). In addition, sialic acid-dependent NK cell inhibition was also observed in a humanized mouse model (55). Macrophage polarization is also influenced by a sialoglycanSiglec pathway $(47,56)$. Alternative M2 polarized macrophages produce cytokines suppressing anti-cancer immunity, secrete pro-angiogenic factors, enhance tumor cell invasion, and thereby promote cancer progression $(60,61)$. Binding of sialylated, cancer-associated MUC1 to Siglec-9 led to a polarization to M2 macrophages in vitro (56). However, studies in Siglec-E deficient mice showed a propensity of Siglec-E deficient macrophages to polarize to M2 macrophages (47). Macrophages express various Siglecs including Siglec-3, Siglec-5/-14, Siglec-7, Siglec9 , and Siglec-10 with some overlapping binding spectra $(7,42-$ 44). The exact function of sialoglycan-Siglec interactions on the influence of pro- and anti-tumorigenic effects of tumorassociated macrophages certainly require further studies. For example, Siglec receptors could also act as potential "don't eat me" signals that inhibit macrophage-mediated phagocytosis (62). Conserved Siglec-15 was identified in a screening of surface markers on antigen-presenting cells that could inhibit $\mathrm{T}$ cell activation (63). Antibodies against Siglec-15 tested in a murine tumor model led to enhanced anti-cancer immunity
(63). Antibodies were humanized and early clinical trials are being planned.

Recent work provided evidence that Siglec receptors are expressed on platelets in both humans and mice $(64,65)$. Engagement of Siglec- 9 or Siglec-E on platelets increased the infectivity of group B streptococci by modulation of platelet activation (64). One could hypothesize that interactions of tumor cell-sialoglycans could also modulate platelet activation and influence metastatic progression.

Two recent studies have found that the sialoglycan-Siglec glyco-immune checkpoint influences activation of tumorinfiltrating lymphocytes (TILs), particularly cytotoxic CD8+ T cells $(51,52)$. We have found that TILs upregulate different inhibitory CD33-related Siglecs, predominantly Siglec-9 in patients with non-small cell lung cancer, colorectal cancer, epithelial ovarian cancer and melanoma (51, 52). Healthy peripheral blood $\mathrm{T}$ cells, however, were not expressing these inhibitory receptors, as described earlier $(51,52)$. Siglec-E was upregulated on tumor-infiltrating $\mathrm{T}$ cells in murine tumor models (51). Inhibition of the sialoglycan-Siglec axis with blocking antibodies or genetic models enhances $\mathrm{T}$ cell-mediated anti-cancer immunity in vitro and in vivo $(51,66,67)$. These results directly implicate that Siglec-9 is a new target that can improve anti-tumoral $\mathrm{T}$ cell activation.

Targeting the sialoglycan-Siglec glyco-immune checkpoint can be achieved by using Siglec-blocking antibodies. Another approach is the reduction of the ligand-density by targeting sialoglycans. Using a sialic acid mimetic that inhibits intratumoral sialoglycan production led to enhanced $\mathrm{T}$ cell-mediated anti-tumor immunity (68). Similar findings were observed with tumor cell lines with defects in sialic acid biosynthesis $(51,69)$. An elegant therapeutic approach is the

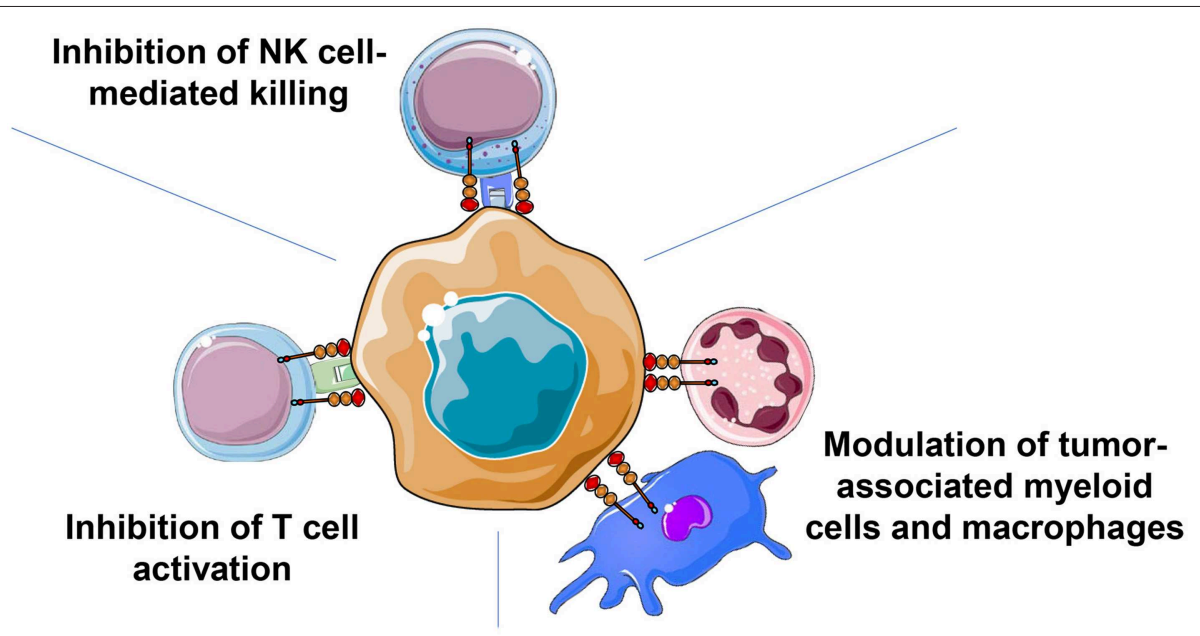

FIGURE 2 | The sialoglycan-Siglec glyco-immune checkpoint involves cells of the innate and the adaptive immune response. Cancer-associated sialoglycans on the surface of tumor cells but also within the tumor microenvironment can mediate immune evasion by engaging Siglec receptors on cells of the innate (NK cells, myeloid cells, and macrophages) and the adaptive (T cells) immune system. Inhibitory Siglec receptors, for example Siglec-9, can inhibit T cell activation by modulating signaling of the T cell receptor. Similarly, NK cell activation and tumor cell killing can be reduced by inhibitory Siglecs such as Siglec-7 and Siglec-9. Interactions of cancer-associated sialoglycans can also regulate myeloid cells and tumor-associated macrophages by influencing the polarization of TAMs and potentially influencing macrophage-mediated phagocytosis via inhibitory Siglec receptors. 
use of sialidases fused to tumor-targeting antibodies that, upon systemic application, mediate hyposialylation of the tumor microenvironment. Xiao et al. have used the anti-HER2 antibody trastuzumab fused with a bacterial sialidase which was shown to increase tumor cell killing in vitro (70) and is currently being tested in pre-clinical mouse models.

\section{INTEGRINS DURING TUMOR CELL DISSEMINATION AND METASTATIC COLONIZATION}

Integrin binding to the components of extracellular matrix (ECM) enables the cell "to sense" the environment and to activate intracellular signaling, which modulates cellular behaviors including survival, proliferation, and migration; thereby sustaining homeostasis. During malignancy, altered expression of integrins together with the loss of cell polarity profoundly changes the cell signaling, which alters oncogenic activity, cell stemness, epithelial plasticity, and angiogenesis [reviewed elsewhere $(2,71,72)$ ].

The integrins comprise a family of heterodimeric $\alpha / \beta$ integrin receptors, which facilitates contacts with components of the extracellular matrix (ECM) and in some cases with adhesion receptors on other cells. A particular integrin receptor with a preference for a specific ligand defines a cell based on the recognition of ECM (e.g., fibronectin, laminin, or collagen). Two receptors, vascular cell adhesion molecule 1 (VCAM1), and intracellular cell adhesion molecules (ICAMs) serve as cell surface receptors for integrins. Integrin-based adhesion of a cell facilitates intracellular adaptor proteins that recruit kinases, for example focal adhesion kinase (FAK) or Src family kinases; and induces signal transduction $(2,72,73)$. Conversely, external signals (growth factors or cytokines) may change the intracellular recruitment of integrins resulting in the modulation of integrin affinity. Integrins can initiate pro-survival but also pro-apoptotic signals (74-76).

A variety of integrins expressed on tumors that originate from epithelial cells, typically facilitate cell adhesion to the basement membrane. Tumor cell surface expression of integrins can vary widely, but is generally associated with the enhanced presence of $\alpha v \beta 3, \alpha v \beta 6, \alpha 5 \beta 1, \alpha 6 \beta 4$ which correlates with the metastatic progression in melanoma, prostate, pancreatic, colon, lung, and breast cancers [reviewed in (73)]. Importantly, integrins within the tumor microenvironment present on endothelial cells, leukocytes, platelets, and other cells of the stroma significantly modulate tumor progression and particularly metastasis.

During the hematogenous phase of metastasis tumor induced platelet activation and the formation of platelet-and fibrinrich tumor cell thrombi (Figure 1), are mediated both by integrins and selectins $(18,77-80)$. In particular, susceptibility to metastasis is associated with tumor cell-derived deposition of certain ECM proteins, such as tenascin C (TNC) in the metastatic niche. TNC is a ligand for $\beta 1$ - and $\beta 3$-integrin and its accumulation in the lungs promotes tumor cell outgrowth and metastasis (81). Osteosarcoma metastasis to the lungs is dependent on TNC expression and the respective expression a receptor on tumor cell $\alpha 9 \beta 1$ integrin (82). The trabecular bone rich in TNC was shown to promote prostate homing of cancer metastasis through $\alpha 9 \beta 1$ integrin (83). Metastatic lung colonization was associated with an induction of stromal periostin expression that is recognized by $\beta 1$ and $\beta 5$ integrins on tumor cells (84).

Integrins also contribute to the formation of a tumor microenvironment. The systemic absence of $\beta 4$ integrin resulted in the attenuation of tumor growth due to impaired angiogenesis (85). Tumor-induced lymphangiogenesis promotes metastasis to the lymph node through the activation of $\alpha 4 \beta 1$ integrin on lymphatic endothelium, which binds to VCAM1-positive tumor cells (86). In addition, myeloid cells expressing $\alpha 4 \beta 1$ integrins accumulate on the tumor-activated endothelium and promote metastasis (87). Antagonist of $\alpha 4 \beta 1$ integrin blocked the recruitment of myeloid cells and thereby angiogenesis and tumor growth. The aberrant expression of VCAM-1 on dormant tumor cells in bone marrow was shown to recruit $\alpha 4 \beta 1$-expressing osteoclast progenitors during bone metastasis (88). It has recently been demonstrated that chemoresistant disseminated tumors occupying the perivascular niche, interacts through $\beta 1$-integrin with VCAM-1 on endothelial cells (89). The inhibition of $\beta 1$ integrin or VCAM-1 sensitizes tumor cells to chemotherapy, making integrin inhibition a viable therapeutic approach to prevent metastasis.

Tumor cell expression of $\alpha v \beta 3$ of $\alpha 4 \beta 1$ integrins is linked to bone metastasis, where they support tumor cell adhesion to ECM proteins such as osteopontin or type I collagen (90). Melanoma cells expressing $\alpha 4 \beta 1$ integrins metastasized to lymph nodes (Figure 1) by binding to VCAM-1 on lymphatic endothelial cells (91). Tumor cell $\alpha_{3} \beta_{1}$ integrin facilitates metastasis by binding to the exposed basement membrane protein laminin5 in the lungs, thereby promoting tumor cell arrest and the onset of outgrowth $(92,93)$. Recently, integrins on tumorderived exosomes were shown to drive the organotropism of tumor metastasis (94). Tumor-derived exosomes carrying a6 34 integrin, target a laminin-rich lung microenvironment, where they induce the accumulation of pro-inflammatory factors required for the promotion of tumor cell seeding and metastasis. While tumor cells do not express leukocyte-specific $\beta 2$ integrins, for example LFA-1, tumor cells that express ICAM-1 facilitate their adherence to leukocytes, particularly neutrophils, through $\beta 2$ integrins, which in turn bind to the endothelium, thereby promoting metastasis $(95,96)$. The role of integrins during cancer is dependent on cues in a tissue context-dependent manner.

Altered glycosylation of integrins on tumor cells modulate the intracellular signaling and cell adhesion (97, 98). An overexpression of branched $\beta 1,6-\mathrm{N}$-acetylglucosamine (GlcNAc) on N-glycans, which is catalyzed by a GnT-V enzyme, is associated with poor prognosis (99), while a knock-down of GnT-V in breast carcinoma cells result in reduced invasiveness (100). Increased branched N-glycans on $\alpha 3 \beta 1$ integrins in B16 mouse melanoma correlated with lung metastasis (101). Galectins are a family of $\beta$-galactoside-binding soluble lectins expressed by tumor cells. The $\mathrm{N}$-glycan on integrin induces the complex formation of $\alpha 6 \beta 4$ integrin/EGFR/ galectin-3 which 
promotes integrin clustering and cell migration and proliferation $(102,103)$. Galectin-3 induced $\alpha v \beta 3$ integrin-mediated clustering was shown to cause tumor growth and drug resistance (104). Mechanistically, unbound $\alpha \mathrm{v} \beta 3$ integrin on tumor cells recruits KRAS to the cell membrane and activates downstream signaling through the NF- $\mathrm{KB}$ pathway and promotes pancreatic carcinoma. In hepatocellular carcinoma metastasis, O-glycosylation of $\beta 1$ integrin influences tumor migration (105). Terminal sialylation on $\mathrm{N}$-glycans and $\mathrm{O}$-glycans of glycoproteins is frequently observed in cancer $(44,59,106)$. Hypersialylation detected in colon, stomach, and ovarian cancers has been linked to an enhanced expression of a2,6-sialyltransferase (ST6Gal-I) and is identified as a marker of poor prognosis (106). In colon carcinoma, enhanced sialylation of $\beta 1$ integrin facilitates adhesion to collagen I and the migration of tumor cells (107). Accordingly, inhibition of ST6Gal-I expression blocks collagen binding and tumor cell migration. Desialylation of O-glycans by sialidase NEU1 suppresses colon-tumor cell adhesion to laminin, tyrosine phosphorylation of integrin $\beta 4$ and metastasis (108). However, in breast cancer cells the $\alpha 2,6$ hypersialylation of $\beta 1$ integrin decreased the adhesion but did not affect invasiveness of these cells (109). These data indicate that glycosylation of integrins modulates adhesion, migration, and signaling of metastatic cells.

\section{CONCLUSIONS AND FUTURE DIRECTIONS}

Alterations in cell adhesion and cell-cell interactions of tumor cells are inherently linked to many processes associated with immune evasion and metastasis that go beyond the scope of this review. The very nature of adhesion receptors on cells to interrogate signals from outside makes the tumor

\section{REFERENCES}

1. Strilic B, Offermanns S. Intravascular survival and extravasation of tumor cells. Cancer Cell. (2017) 32:282-93. doi: 10.1016/j.ccell.2017.07.001

2. Cooper J, Giancotti FG. Integrin signaling in cancer: mechanotransduction, stemness, epithelial plasticity, and therapeutic resistance. Cancer Cell. (2019) 35:347-67. doi: 10.1016/j.ccell.2019.01.007

3. Vajaria BN, Patel PS. Glycosylation: a hallmark of cancer? Glycoconj J. (2017) 34:147-156. doi: 10.1007/s10719-016-9755-2

4. Hauselmann I, Borsig L. Altered tumor-cell glycosylation promotes metastasis. Front Oncol. (2014) 4:28. doi: 10.3389/fonc.2014.00028

5. Fuster MM, Esko JD. The sweet and sour of cancer: glycans as novel therapeutic targets. Nat Rev Cancer. (2005) 5:526-42. doi: 10.1038/nrc 1649

6. Rodrigues JG, Balmana M, Macedo JA, Pocas J, Fernandes A, de-FreitasJunior JCM, et al. Glycosylation in cancer: selected roles in tumour progression, immune modulation and metastasis. Cell Immunol. (2018) 333:46-57. doi: 10.1016/j.cellimm.2018.03.007

7. Adams OJ, Stanczak MA, von Gunten S, Laubli H. Targeting sialic acid-Siglec interactions to reverse immune suppression in cancer. Glycobiology. (2018) 28:640-7. doi: 10.1093/glycob/cwx108

8. Reymond N, d'Agua BB, Ridley AJ. Crossing the endothelial barrier during metastasis. Nat Rev Cancer. (2013) 13:858-70. doi: 10.1038/nrc3628

9. Ley K. The role of selectins in inflammation and disease. Trends Mol Med. (2003) 9:263-8. doi: 10.1016/S1471-4914(03)00071-6 microenvironment a crucial factor during immune evasion and metastasis. The biology of cell adhesion during cancer progression remains complex mainly due to: (a) several receptors likely act in parallel during any metastasis; (b) cell surface changes are linked to tumor heterogeneity; (c) diverse tumor glycosylation affect both receptors and ligands. Nevertheless, the potential to target cell adhesion mechanisms for tumor therapies is continuously being explored. For instance, fusion of a IL-2 cytokine with an Fc part of an antibody targeting RGD sequence of an integrin demonstrated promising results in mouse models when applied in combination with a PD-1 checkpoint blockade inhibitor (110). Another study has demonstrated that fucosylated nanoparticles can target irradiation-activated vasculature of the tumor, associated with enhanced P-selectin expression (111). Altered tumor glycosylation is a common culprit that contributes to tumor cell dissemination and immune suppression. Thus, further efforts to "edit" the tumor glycosylation landscape using sialidase and thereby changing the Siglec immune responsiveness or metastasis holds great potential in clinical applications (70). Nevertheless, further understanding of the tumor microenvironment is a prerequisite for designing an intervention on cell adhesion mechanisms that will be likely used in combination either with standard- or immune-therapy.

\section{AUTHOR CONTRIBUTIONS}

All authors listed have made a substantial, direct and intellectual contribution to the work, and approved it for publication.

\section{FUNDING}

This work was supported by the SNF grant \#310030-173076 to LB and \#310030-184720 to HL.
10. McEver RP. Selectins: lectins that initiate cell adhesion under flow. Curr Opin Cell Biol. (2002) 14:581-6. doi: 10.1016/S0955-0674(02)00367-8

11. Borsig L. Selectins in cancer immunity. Glycobiology. (2018) 28:648-55. doi: $10.1093 /$ glycob/cwx105

12. Läubli H, Borsig L. Selectins as mediators of lung metastasis. Cancer Microenviron. (2010) 3:97-105. doi: 10.1007/s12307-010-0043-6

13. Varki A. Selectin ligands: will the real ones please stand up? J Clin Invest. (1997) 99:158-62. doi: 10.1172/JCI119142

14. Läubli H, and Borsig L. Selectins promote tumor metastasis. Semin Cancer Biol. (2010) 20:169-77. doi: 10.1016/j.semcancer.2010.04.005

15. Kannagi R. Molecular mechanism for cancer-associated induction of sialyl Lewis $\mathrm{X}$ and sialyl Lewis A expression-The Warburg effect revisited. Glycoconj J. (2004) 20:353-64. doi: 10.1023/B:GLYC.0000033631. 35357.41

16. Hollingsworth MA, Swanson BJ. Mucins in cancer: protection and control of the cell surface. Nat Rev Cancer. (2004) 4:45-60. doi: 10.1038/nrc1251

17. Szczerba BM, Castro-Giner F, Vetter M, Krol I, Gkountela S, Landin J, et al. Neutrophils escort circulating tumour cells to enable cell cycle progression. Nature. (2019) 566:553-7. doi: 10.1038/s41586-019-0915-y

18. Nieswandt B, Hafner M, Echtenacher B, Mannel DN. Lysis of tumor cells by natural killer cells in mice is impeded by platelets. Cancer Res. (1999) 59:1295-300.

19. Labelle M, Begum S, Hynes RO. Platelets guide the formation of early metastatic niches. Proc Natl Acad Sci USA. (2014) 111:E3053-61. doi: $10.1073 /$ pnas. 1411082111 
20. Borsig L, Wong R, Feramisco J, Nadeau DR, Varki NM, Varki A. Heparin and cancer revisited: mechanistic connections involving platelets, P-selectin, carcinoma mucins, and tumor metastasis. Proc Natl Acad Sci USA. (2001) 98:3352-7. doi: 10.1073/pnas.061615598

21. Gerdes N, Seijkens T, Lievens D, Kuijpers MJ, Winkels H, Projahn D, et al. Platelet CD40 exacerbates atherosclerosis by transcellular activation of endothelial cells and leukocytes. Arterioscler Thromb Vasc Biol. (2016) 36:482-90. doi: 10.1161/ATVBAHA.115.307074

22. Ingersoll SB, Langer F, Walker JM, Meyer T, Robson T, Amaya M, et al. Deficiencies in the CD40 and CD154 receptor-ligand system reduce experimental lung metastasis. Clin Exp Metastasis. (2009) 26:829-37. doi: 10.1007/s10585-009-9282-7

23. Läubli H, Spanaus KS, Borsig L. Selectin-mediated activation of endothelial cells induces expression of CCL5 and promotes metastasis through recruitment of monocytes. Blood. (2009) 114:4583-91. doi: 10.1182/blood-2008-10-186585

24. Borsig L, Wolf MJ, Roblek M, Lorentzen A, Heikenwalder M. Inflammatory chemokines and metastasis-tracing the accessory. Oncogene. (2014) 33:321724. doi: $10.1038 /$ onc. 2013.272

25. Gout S, Tremblay PL, Huot J. Selectins and selectin ligands in extravasation of cancer cells and organ selectivity of metastasis. Clin Exp Metastasis. (2008) 25:335-44. doi: 10.1007/s10585-007-9096-4

26. St Hill CA. Interactions between endothelial selectins and cancer cells regulate metastasis. Front Biosci. (2011) 17: 3909. doi: 10.2741/3909

27. Hanley WD, Burdick MM, Konstantopoulos K, Sackstein R. CD44 on LS174T colon carcinoma cells possesses E-selectin ligand activity. Cancer Res. (2005) 65:5812-7. doi: 10.1158/0008-5472.CAN-04-4557

28. Hauselmann I, Roblek M, Protsyuk D, Huck V, Knopfova L, Grassle $\mathrm{S}$, et al. Monocyte induction of E-selectin-mediated endothelial activation releases VE-cadherin junctions to promote tumor cell extravasation in the metastasis cascade. Cancer Res. (2016) 76:5302-12. doi: 10.1158/0008-5472.CAN-16-0784

29. Esposito M, Mondal N, Greco TM, Wei Y, Spadazzi C, Lin SC, et al. Bone vascular niche E-selectin induces mesenchymal-epithelial transition and Wnt activation in cancer cells to promote bone metastasis. Nat Cell Biol. (2019) 21:627-39. doi: 10.1038/s41556-019-0309-2

30. Tremblay PL, Auger FA, Huot J. Regulation of transendothelial migration of colon cancer cells by E-selectin-mediated activation of p38 and ERK MAP kinases. Oncogene. (2006) 25:6563-73. doi: 10.1038/sj.onc.12 09664

31. Läubli H, Stevenson JL, Varki A, Varki NM, Borsig L. L-selectin facilitation of metastasis involves temporal induction of fut7-dependent ligands at sites of tumor cell arrest. Cancer Res. (2006) 66:1536-42. doi: 10.1158/0008-5472.CAN-05-3121

32. Borsig L, Wong R, Hynes RO, Varki NM, Varki A. Synergistic effects of Land $\mathrm{P}$-selectin in facilitating tumor metastasis can involve non-mucin ligands and implicate leukocytes as enhancers of metastasis. Proc Natl Acad Sci USA. (2002) 99:2193-8. doi: 10.1073/pnas.261704098

33. Wolf MJ, Hoos A, Bauer J, Boettcher S, Knust M, Weber A, et al. Endothelial CCR2 signaling induced by colon carcinoma cells enables extravasation via the JAK2-Stat5 and p38MAPK pathway. Cancer Cell. (2012) 22:91-105. doi: 10.1016/j.ccr.2012.05.023

34. Hoos A, Protsyuk D, Borsig L. Metastatic growth progression caused by PSGL-1-mediated recruitment of monocytes to metastatic sites. Cancer Res. (2014) 74:695-704. doi: 10.1158/0008-5472.CAN-13-0946

35. Venturi GM, Tu L, Kadono T, Khan AI, Fujimoto Y, Oshel P, et al. Leukocyte migration is regulated by L-selectin endoproteolytic release. Immunity. (2003) 19:713-24. doi: 10.1016/S1074-7613(03)00295-4

36. Galkina E, Tanousis K, Preece G, Tolaini M, Kioussis D, Florey O, et al. Lselectin shedding does not regulate constitutive $\mathrm{T}$ cell trafficking but controls the migration pathways of antigen-activated T lymphocytes. J Exp Med. (2003) 198:1323-35. doi: 10.1084/jem.20030485

37. Gattinoni L, Klebanoff CA, Palmer DC, Wrzesinski C, Kerstann K, Yu Z, et al. Acquisition of full effector function in vitro paradoxically impairs the in vivo antitumor efficacy of adoptively transferred CD8+ T cells. J Clin Invest. (2005) 115:1616-26. doi: 10.1172/JCI24480

38. Klebanoff CA, Gattinoni L, Torabi-Parizi P, Kerstann K, Cardones AR, Finkelstein SE, et al. Central memory self/tumor-reactive CD8 $+\mathrm{T}$ cells confer superior antitumor immunity compared with effector memory T cells Proc Natl Acad Sci USA. (2005) 102:9571-6. doi: 10.1073/pnas.0503726102

39. Tian G, Courtney AN, Jena B, Heczey A, Liu D, Marinova E, et al. CD62L+ NKT cells have prolonged persistence and antitumor activity in vivo. J Clin Invest. (2016) 126:2341-55. doi: 10.1172/JCI83476

40. Watson HA, Durairaj RRP, Ohme J, Alatsatianos M, Almutairi $\mathrm{H}$, Mohammed RN, et al. L-selectin enhanced $\mathrm{T}$ cells improve the efficacy of cancer immunotherapy. Front Immunol. (2019) 10:1321. doi: 10.3389/fimmu.2019.01321

41. Varki A, Schnaar RL, Schauer R. Sialic acids and other nonulosonic acids. In: Varki A, Cummings RD, Esko JD, Stanley P, Hart GW, Aebi M, Darvill AG, Kinoshita T, Packer NH, Prestegard JH, Schnaar RL and Seeberger PH, editors. Essentials of Glycobiology. New York, NY: Cold Spring Harbor (2015). p. 179-95.

42. Crocker PR, Paulson JC, Varki A. Siglecs and their roles in the immune system. Nat Rev Immunol. (2007) 7:255-66. doi: 10.1038/nri2056

43. Fraschilla I, Pillai S. Viewing Siglecs through the lens of tumor immunology. Immunol Rev. (2017) 276:178-91. doi: 10.1111/imr.12526

44. Rodrigues E, Macauley MS. Hypersialylation in cancer: modulation of inflammation and therapeutic opportunities. Cancers. (2018) 10:E207. doi: 10.3390/cancers10060207

45. Schwarz F, Fong JJ, Varki A. Human-specific evolutionary changes in the biology of siglecs. Adv Exp Med Biol. (2015) 842:1-16. doi: 10.1007/978-3-319-11280-0_1

46. Angata T. Possible influences of endogenous and exogenous ligands on the evolution of human Siglecs. Front Immunol. (2018) 9:2885. doi: 10.3389/fimmu.2018.02885

47. Laubli H, Pearce OM, Schwarz F, Siddiqui SS, Deng L, Stanczak MA, et al. Engagement of myelomonocytic Siglecs by tumor-associated ligands modulates the innate immune response to cancer. Proc Natl Acad Sci USA. (2014) 111:14211-6. doi: 10.1073/pnas.1409580111

48. Padler-Karavani V, Hurtado-Ziola N, Chang YC, Sonnenburg JL, Ronaghy A, $\mathrm{Yu} \mathrm{H}$, et al. Rapid evolution of binding specificities and expression patterns of inhibitory CD33-related Siglecs in primates. FASEB J. (2014) 28:1280-93. doi: 10.1096/fj.13-241497

49. Propster JM, Yang F, Rabbani S, Ernst B, Allain FH, Schubert M. Structural basis for sulfation-dependent self-glycan recognition by the human immuneinhibitory receptor Siglec-8. Proc Natl Acad Sci USA. (2016) 113:E4170-9. doi: 10.1073/pnas.1602214113

50. Bochner BS, Alvarez RA, Mehta P, Bovin NV, Blixt O, White JR, et al. Glycan array screening reveals a candidate ligand for Siglec-8. J Biol Chem. (2005) 280:4307-12. doi: 10.1074/jbc.M412378200

51. Stanczak MA, Siddiqui SS, Trefny MP, Thommen DS, Boligan KF, von Gunten S, et al. Self-associated molecular patterns mediate cancer immune evasion by engaging Siglecs on T cells. J Clin Invest. (2018) 128:4912-23. doi: 10.1172/JCI120612

52. Haas Q, Boligan KF, Jandus C, Schneider C, Simillion C, Stanczak MA, et al. Siglec-9 regulates an effector memory CD8 $(+)$ T-cell subset that congregates in the melanoma tumor microenvironment. Cancer Immunol Res. (2019) 7:707-18. doi: 10.1158/2326-6066.CIR-18-0505

53. Laubli H, Alisson-Silva F, Stanczak MA, Siddiqui SS, Deng L, Verhagen A, et al. Lectin galactoside-binding soluble 3 binding protein (LGALS3BP) is a tumor-associated immunomodulatory ligand for CD33-related Siglecs. J Biol Chem. (2014) 289:33481-91. doi: 10.1074/jbc.M114.5 93129

54. Hudak JE, Canham SM, Bertozzi CR. Glycocalyx engineering reveals a Siglec-based mechanism for NK cell immunoevasion. Nat Chem Biol. (2014) 10:69-75. doi: 10.1038/nchembio. 1388

55. Jandus C, Boligan KF, Chijioke O, Liu H, Dahlhaus M, Demoulins T, et al. Interactions between Siglec-7/9 receptors and ligands influence NK celldependent tumor immunosurveillance. J Clin Invest. (2014) 124:1810-20. doi: 10.1172/JCI65899

56. Beatson R, Tajadura-Ortega V, Achkova D, Picco G, Tsourouktsoglou TD, Klausing S, et al. The mucin MUC1 modulates the tumor immunological microenvironment through engagement of the lectin Siglec-9. Nat Immunol. (2016) 17:1273-81. doi: 10.1038/ni.3552

57. Boligan KF, Mesa C, Fernandez LE, von Gunten S. Cancer intelligence acquired (CIA): tumor glycosylation and sialylation codes 
dismantling antitumor defense. Cell Mol Life Sci. (2015) 72:1231-48. doi: 10.1007/s00018-014-1799-5

58. Haas Q, Simillion C, von Gunten S. A cartography of siglecs and sialyltransferases in gynecologic malignancies: is there a road towards a sweet future? Front Oncol. (2018) 8:68. doi: 10.3389/fonc.2018.00068

59. Pearce OM, Laubli H. Sialic acids in cancer biology and immunity. Glycobiology. (2016) 26:111-28. doi: 10.1093/glycob/cwv097

60. Mantovani A, Marchesi F, Malesci A, Laghi L, Allavena P. Tumour-associated macrophages as treatment targets in oncology. Nat Rev Clin Oncol. (2017) 14:399-416. doi: 10.1038/nrclinonc.2016.217

61. De Palma M, Lewis CE. Macrophage regulation of tumor responses to anticancer therapies. Cancer Cell. (2013) 23:277-86. doi: 10.1016/j.ccr.2013.02.013

62. Barkal AA, Brewer RE, Markovic M, Kowarsky M, Barkal SA, Zaro BW, et al. CD24 signalling through macrophage Siglec-10 is a target for cancer immunotherapy. Nature. (2019) 572:392-6. doi: 10.1038/s41586-019-1456-0

63. Wang J, Sun J, Liu LN, Flies DB, Nie X, Toki M, et al. Siglec-15 as an immune suppressor and potential target for normalization cancer immunotherapy. Nat Med. (2019) 25:656-66. doi: 10.1038/s41591-019-0374-x

64. Uchiyama S, Sun J, Fukahori K, Ando N, Wu M, Schwarz F, et al. Dual actions of group B Streptococcus capsular sialic acid provide resistance to plateletmediated antimicrobial killing. Proc Natl Acad Sci USA. (2019) 116:7465-70. doi: $10.1073 /$ pnas. 1815572116

65. Nguyen KA, Hamzeh-Cognasse H, Palle S, Anselme-Bertrand I, Arthaud CA, Chavarin P, et al. Role of Siglec-7 in apoptosis in human platelets. PLoS ONE. (2014) 9:e106239. doi: 10.1371/journal.pone.0106239

66. Ikehara Y, Ikehara SK, Paulson JC. Negative regulation of T cell receptor signaling by Siglec-7 (p70/AIRM) and Siglec-9. J Biol Chem. (2004) 279:43117-25. doi: 10.1074/jbc.M403538200

67. Nguyen DH, Hurtado-Ziola N, Gagneux P, Varki A. Loss of Siglec expression on T lymphocytes during human evolution. Proc Natl Acad Sci USA. (2006) 103:7765-70. doi: 10.1073/pnas.0510484103

68. Bull C, Boltje TJ, Balneger N, Weischer SM, Wassink M, van Gemst JJ, et al. Sialic acid blockade suppresses tumor growth by enhancing T-cell-mediated tumor immunity. Cancer Res. (2018) 78:3574-88. doi: 10.1158/0008-5472.CAN-17-3376

69. Perdicchio M, Cornelissen LA, Streng-Ouwehand I, Engels S, Verstege MI, Boon L, et al. Tumor sialylation impedes $\mathrm{T}$ cell mediated anti-tumor responses while promoting tumor associated-regulatory T cells. Oncotarget. (2016) 7:8771-82. doi: 10.18632/oncotarget.6822

70. Xiao H, Woods EC, Vukojicic P, Bertozzi CR. Precision glycocalyx editing as a strategy for cancer immunotherapy. Proc Natl Acad Sci USA. (2016) 113:10304-9. doi: 10.1073/pnas.1608069113

71. Hynes RO. Integrins: versatility, modulation, and signaling in cell adhesion. Cell. (1992) 69:11-25. doi: 10.1016/0092-8674(92)90115-S

72. Seguin L, Desgrosellier JS, Weis SM, Cheresh DA. Integrins and cancer: regulators of cancer stemness, metastasis, and drug resistance. Trends Cell Biol. (2015) 25:234-40. doi: 10.1016/j.tcb.2014.12.006

73. Desgrosellier JS, Cheresh DA. Integrins in cancer: biological implications and therapeutic opportunities. Nat Rev Cancer. (2010) 10:9-22. doi: $10.1038 / \mathrm{nrc} 2748$

74. Aoudjit F, Vuori K. Integrin signaling inhibits paclitaxel-induced apoptosis in breast cancer cells. Oncogene. (2001) 20:4995-5004. doi: 10.1038/sj.onc.1204554

75. Scatena M, Almeida M, Chaisson ML, Fausto N, Nicosia RF, Giachelli CM. NF-kappaB mediates alphavbeta3 integrin-induced endothelial cell survival. J Cell Biol. (1998) 141:1083-93. doi: 10.1083/jcb.141.4.1083

76. Stupack DG, Puente XS, Boutsaboualoy S, Storgard CM, Cheresh DA. Apoptosis of adherent cells by recruitment of caspase-8 to unligated integrins. J Cell Biol. (2001) 155:459-70. doi: 10.1083/jcb.200106070

77. Labelle M, Begum S, Hynes RO. Direct signaling between platelets and cancer cells induces an epithelial-mesenchymal-like transition and promotes metastasis. Cancer Cell. (2011) 20:576-90. doi: 10.1016/j.ccr.2011.09.009

78. Kim YJ, Borsig L, Varki NM, Varki A. P-selectin deficiency attenuates tumor growth and metastasis. Proc Natl Acad Sci USA. (1998) 95:9325-30. doi: $10.1073 /$ pnas. 95.16 .9325

79. Felding-Habermann B, O'Toole TE, Smith JW, Fransvea E, Ruggeri $\mathrm{ZM}$, Ginsberg $\mathrm{MH}$, et al. Integrin activation controls metastasis in human breast cancer. Proc Natl Acad Sci USA. (2001) 98:1853-8. doi: 10.1073/pnas.98.4.1853

80. Trikha M, Zhou Z, Timar J, Raso E, Kennel M, Emmell E, et al. Multiple roles for platelet GPIIb/IIIa and alphavbeta3 integrins in tumor growth, angiogenesis, and metastasis. Cancer Res. (2002) 62:2824-33. Available online at: https://cancerres.aacrjournals.org/content/62/10/2824

81. Oskarsson T, Acharyya S, Zhang XH, Vanharanta S, Tavazoie SF, Morris PG, et al. Breast cancer cells produce tenascin $\mathrm{C}$ as a metastatic niche component to colonize the lungs. Nat Med. (2011) 17:867-74. doi: 10.1038/nm. 2379

82. Sun Z, Schwenzer A, Rupp T, Murdamoothoo D, Vegliante R, Lefebvre O, et al. Tenascin-C promotes tumor cell migration and metastasis through integrin alpha9beta1-mediated YAP inhibition. Cancer Res. (2018) 78:95061. doi: 10.1158/0008-5472.CAN-17-1597

83. San Martin R, Pathak R, Jain A, Jung SY, Hilsenbeck SG, Pina-Barba $\mathrm{MC}$, et al. Tenascin-C and integrin alpha9 mediate interactions of prostate cancer with the bone microenvironment. Cancer Res. (2017) 77:5977-88. doi: 10.1158/0008-5472.CAN-17-0064

84. Malanchi I, Santamaria-Martinez A, Susanto E, Peng H, Lehr HA, Delaloye JF, et al. Interactions between cancer stem cells and their niche govern metastatic colonization. Nature. (2012) 481:85-9. doi: 10.1038/nature 10694

85. Nikolopoulos SN, Blaikie P, Yoshioka T, Guo W, Giancotti FG. Integrin beta4 signaling promotes tumor angiogenesis. Cancer Cell. (2004) 6:471-83. doi: 10.1016/j.ccr.2004.09.029

86. Garmy-Susini B, Avraamides CJ, Desgrosellier JS, Schmid MC, Foubert P, Ellies LG, et al. PI3Kalpha activates integrin alpha4betal to establish a metastatic niche in lymph nodes. Proc Natl Acad Sci USA. (2013) 110:9042-7. doi: 10.1073/pnas.1219603110

87. Jin H, Aiyer A, Su J, Borgstrom P, Stupack D, Friedlander M, et al. A homing mechanism for bone marrow-derived progenitor cell recruitment to the neovasculature. J Clin Invest. (2006) 116:652-62. doi: 10.1172/JCI24751

88. Lu X, Mu E, Wei Y, Riethdorf S, Yang Q, Yuan M, et al. VCAM-1 promotes osteolytic expansion of indolent bone micrometastasis of breast cancer by engaging alpha4betal-positive osteoclast progenitors. Cancer cell. (2011) 20:701-14. doi: 10.1016/j.ccr.2011.11.002

89. Carlson P, Dasgupta A, Grzelak CA, Kim J, Barrett A, Coleman IM, et al. Targeting the perivascular niche sensitizes disseminated tumour cells to chemotherapy. Nat Cell Biol. (2019) 21:238-50. doi: 10.1038/s41556-018-0267-0

90. Cooper CR, Chay CH, Pienta KJ. The role of alpha(v)beta(3) in prostate cancer progression. Neoplasia. (2002) 4:191-4. doi: 10.1038/sj.neo.7900224

91. Schlesinger M, Bendas G. Contribution of very late antigen-4 (VLA-4) integrin to cancer progression and metastasis. Cancer Metastasis Rev. (2015) 34:575-91. doi: 10.1007/s10555-014-9545-x

92. Wang $\mathrm{H}, \mathrm{Fu} \mathrm{W}, \mathrm{Im} \mathrm{JH}$, Zhou Z, Santoro SA, Iyer V, et al. Tumor cell alpha3beta1 integrin and vascular laminin-5 mediate pulmonary arrest and metastasis. J Cell Biol. (2004) 164:935-41. doi: 10.1083/jcb.200309112

93. Shibue T, Weinberg RA. Integrin betal-focal adhesion kinase signaling directs the proliferation of metastatic cancer cells disseminated in the lungs. Proc Natl Acad Sci USA. (2009) 106:10290-5. doi: 10.1073/pnas.0904227106

94. Hoshino A, Costa-Silva B, Shen TL, Rodrigues G, Hashimoto A, Tesic Mark $\mathrm{M}$, et al. Tumour exosome integrins determine organotropic metastasis. Nature. (2015) 527:329-35. doi: 10.1038/nature15756

95. Strell C, Entschladen F. Extravasation of leukocytes in comparison to tumor cells. Cell Commun Signal. (2008) 6:10. doi: 10.1186/1478-811X-6-10

96. Huh SJ, Liang S, Sharma A, Dong C, Robertson GP. Transiently entrapped circulating tumor cells interact with neutrophils to facilitate lung metastasis development. Cancer Res. (2010) 70:6071-82. doi: 10.1158/0008-5472.CAN-09-4442

97. Marsico G, Russo L, Quondamatteo F, Pandit A. Glycosylation and integrin regulation in cancer. Trends Cancer. (2018) 4:537-52. doi: 10.1016/j.trecan.2018.05.009

98. Kariya Y, Kariya Y, Gu J. Roles of integrin $\alpha 6 \beta 4$ Glycosylation in cancer. Cancer. (2017) 9:79. doi: 10.3390/cancers 9070079

99. Dennis JW, Laferte S, Waghorne C, Breitman ML, Kerbel RS. Beta 16 branching of Asn-linked oligosaccharides is directly associated with metastasis. Science. (1987) 236:582-5. doi: 10.1126/science.2953071 
100. Guo HB, Randolph M, Pierce M. Inhibition of a specific N-glycosylation activity results in attenuation of breast carcinoma cell invasivenessrelated phenotypes: inhibition of epidermal growth factor-induced dephosphorylation of focal adhesion kinase. J Biol Chem. (2007) 282:22150-62. doi: 10.1074/jbc.M611518200

101. Yoshimura M, Nishikawa A, Ihara Y, Taniguchi S, Taniguchi N. Suppression of lung metastasis of B16 mouse melanoma by $\mathrm{N}$ acetylglucosaminyltransferase III gene transfection. Proc Natl Acad Sci USA. (1995) 92:8754-8. doi: 10.1073/pnas.92.19.8754

102. Hang Q, Isaji T, Hou S, Zhou Y, Fukuda T, Gu J. N-Glycosylation of integrin $\alpha 5$ acts as a switch for EGFR-mediated complex formation of integrin $\alpha 5 \beta 1$ to $\alpha 6 \beta 4$. Sci Rep. (2016) 6:33507. doi: 10.1038/srep33507

103. Kariya Y, Oyama M, Hashimoto Y, Gu J, Kariya Y. $\beta 4$-integrin/PI3K signaling promotes tumor progression through the galectin-3-N-glycan complex. Mol Cancer Res. (2018) 16:1024-34. doi: 10.1158/1541-7786.MCR-17-0365

104. Seguin L, Kato S, Franovic A, Camargo MF, Lesperance J, Elliott KC, et al. An integrin $\beta$ (3)-KRAS-RalB complex drives tumour stemness and resistance to EGFR inhibition. Nat Cell Biol. (2014) 16:457-68. doi: 10.1038/ncb2953

105. Liu $\mathrm{CH}, \mathrm{Hu} \mathrm{RH}$, Huang MJ, Lai IR, Chen $\mathrm{CH}$, Lai HS, et al. C1GALT1 promotes invasive phenotypes of hepatocellular carcinoma cells by modulating integrin betal glycosylation and activity. PLoS ONE. (2014) 9:e94995. doi: 10.1371/journal.pone.0094995

106. Pinho SS, Reis CA. Glycosylation in cancer: mechanisms and clinical implications. Nat Rev Cancer. (2015) 15:540-55. doi: 10.1038/nrc3982

107. Seales EC, Jurado GA, Brunson BA, Wakefield JK, Frost AR, Bellis SL. Hypersialylation of betal integrins, observed in colon adenocarcinoma, may contribute to cancer progression by up-regulating cell motility. Cancer Res. (2005) 65:4645-52. doi: 10.1158/0008-5472.CAN-04-3117
108. Uemura T, Shiozaki K, Yamaguchi K, Miyazaki S, Satomi S, Kato K, et al Contribution of sialidase NEU1 to suppression of metastasis of human colon cancer cells through desialylation of integrin beta4. Oncogene. (2009) 28:1218-29. doi: 10.1038/onc.2008.471

109. Yuan Y, Wu L, Shen S, Wu S, Burdick MM. Effect of alpha 2,6 sialylation on integrin-mediated adhesion of breast cancer cells to fibronectin and collagen I. Life Sci. (2016) 149:138-45. doi: 10.1016/j.lfs.2016.02.071

110. Kwan BH, Zhu EF, Tzeng A, Sugito HR, Eltahir AA, Ma B, et al. Integrin-targeted cancer immunotherapy elicits protective adaptive immune responses. J Exp Med. (2017) 214:1679-90. doi: 10.1084/jem.20160831

111. Shamay Y, Elkabets M, Li H, Shah J, Brook S, Wang F, et al. P-selectin is a nanotherapeutic delivery target in the tumor microenvironment. Sci Transl Med. (2016) 8:345ra87. doi: 10.1126/scitranslmed.aaf7374

Conflict of Interest Statement: HL received travel grants and consultant fees from Bristol-Myers Squibb (BMS) and Merck, Sharp, and Dohme (MSD), and Roche. HL received research support from BMS and Palleon Pharmaceuticals.

The remaining author declares that the research was conducted in the absence of any commercial or financial relationships that could be construed as a potential conflict of interest.

Copyright (c) 2019 Läubli and Borsig. This is an open-access article distributed under the terms of the Creative Commons Attribution License (CC BY). The use, distribution or reproduction in other forums is permitted, provided the original author(s) and the copyright owner(s) are credited and that the original publication in this journal is cited, in accordance with accepted academic practice. No use, distribution or reproduction is permitted which does not comply with these terms. 